\title{
Daily variation at three Antarctic geomagnetic observatories within the polar cap
}

\author{
M. Pietrolungo, S. Lepidi, L. Cafarella, L. Santarelli, and D. Di Mauro \\ Istituto Nazionale di Geofisica e Vulcanologia, Roma, Italy \\ Received: 16 November 2007 - Revised: 2 June 2008 - Accepted: 25 June 2008 - Published: 4 August 2008
}

\begin{abstract}
In this work we present a statistical analysis of the diurnal variation as observed at three Antarctic observatories located at different positions within the polar cap during the year 2006. Data used for the analysis are from the Italian geomagnetic observatory at Mario Zucchelli Station (formerly Terra Nova Bay, geographic latitude $74.7^{\circ} \mathrm{S}$, corrected geomagnetic latitude $80.0^{\circ} \mathrm{S}$ ), from the French-Italian observatory at Concordia Station $\left(75.1^{\circ} \mathrm{S}, 88.9^{\circ} \mathrm{S}\right)$ and from the French observatory at Dumont D'Urville $\left(66.7^{\circ} \mathrm{S}, 80.4^{\circ} \mathrm{S}\right)$, which are located in pairs at the same geographic and corrected geomagnetic latitude; such a position allows to distinguish whether the geographic or the geomagnetic reference system is better suitable to describe the observed phenomena at so high latitudes. The peculiarities of the daily variation as observed during this year and its relation with the observatory location and magnetospheric and interplanetary conditions were analysed. Data were also studied taking into account different Lloyd seasons. The results indicate that the 24-h variation is quite persistent, but its amplitude strongly depends on season and global geomagnetic activity: indeed, it almost vanishes during local winter for quiet geomagnetic conditions; this reduction is more evident at the stations closer to the geographic pole, where the solar radiation reduction during winter is more dramatic. The Interplanetary Magnetic Field orientation has been found to be important in that the north-south and the east-west components control the amplitude and the diurnal pattern of the variation, respectively.
\end{abstract}

Keywords. Geomagnetism and paleomagnetism (Time variations, diurnal to secular) - Magnetospheric physics (Polar cap phenomena; Solar wind-magnetosphere interactions)

Correspondence to: M. Pietrolungo

(pietrolungo@ingv.it)

\section{Introduction}

It is well known that the mid latitude daily variation field ( $24 \mathrm{~h}$ period and its harmonics), called geomagnetic $S_{q}$ field in short, is mainly due to the ionospheric currents generated by the dynamo action of the neutral winds in the ionosphere interacting with the geomagnetic main field. The daily variation in the polar cap is generated by two different current systems: the $S_{q}$ and the polar cap electric current system $S_{q}^{p}$, related to field aligned currents that are linked to processes in the magnetosphere and its interaction with the Interplanetary Magnetic Field (IMF) (e.g. Akasofu, 1983; Ratcliffe, 1972; Kivelson and Russel, 1996; Brekke, 1997). The analysis of the geomagnetic data collected in polar observatories is very useful in order to improve our comprehension of these complex and fascinating phenomena.

In a previous paper (Santarelli et al., 2007), we analyzed the daily variation as observed at the Italian Antarctic geomagnetic observatory at Mario Zucchelli Station (IAGA code TNB) during fourteen years (1987-2000); we found a pronounced seasonal effect in the amplitude of the diurnal variation, but not in its pattern. We also studied the daily variation at the French-Italian Antarctic geomagnetic observatory at Concordia Station (IAGA code DMC) for a period of about one month (a preliminary test campaign conducted during the 1999-2000 summer expedition; Lepidi et al., 2003) and during the years 2005 and 2006, taking into account different seasons and different magnetospheric and interplanetary magnetic field conditions (Cafarella et al., 2007). The results showed that the 24-h variation at this observatory exhibits a pronounced day-to-day amplitude variability related to the global magnetospheric activity level. It was also shown that different IMF conditions correspond to different electric current contributions to daily variation and to a time shift. In this paper an extension of this last work is presented; it takes into account data from three instead of one Antarctic observatory, all located within the polar cap.

Published by Copernicus Publications on behalf of the European Geosciences Union. 
Table 1. Geographic and corrected geomagnetic (IGRF06) coordinates, declination $D$ (average value from the 2006 experimental data), geographic local time (LT) and corrected geomagnetic local time (MLT) of the three stations TNB, DRV and DMC; the last column reports the year from which data are available.

\begin{tabular}{ccccccc}
\hline Station & Geogr. Coord. & Geom. Coord. & $D$ & LT & MLT & Data from \\
\hline TNB & $74.7^{\circ} \mathrm{S} 164.1^{\circ} \mathrm{E}$ & $80.0^{\circ} \mathrm{S} 306.8^{\circ} \mathrm{E}$ & $136^{\circ}$ & $\mathrm{UT}+10: 56$ & $\mathrm{UT}-08: 13$ & 1987 \\
DRV & $66.7^{\circ} \mathrm{S} 140.0^{\circ} \mathrm{E}$ & $80.4^{\circ} \mathrm{S} 236.0^{\circ} \mathrm{E}$ & $190^{\circ}$ & $\mathrm{UT}+9: 20$ & $\mathrm{UT}-12: 54$ & 1957 \\
DMC & $75.1^{\circ} \mathrm{S} 123.4^{\circ} \mathrm{E}$ & $88.9^{\circ} \mathrm{S} 54.3^{\circ} \mathrm{E}$ & $222^{\circ}$ & $\mathrm{UT}+8: 14$ & $\mathrm{UT}-01: 01$ & 2005 \\
\hline
\end{tabular}

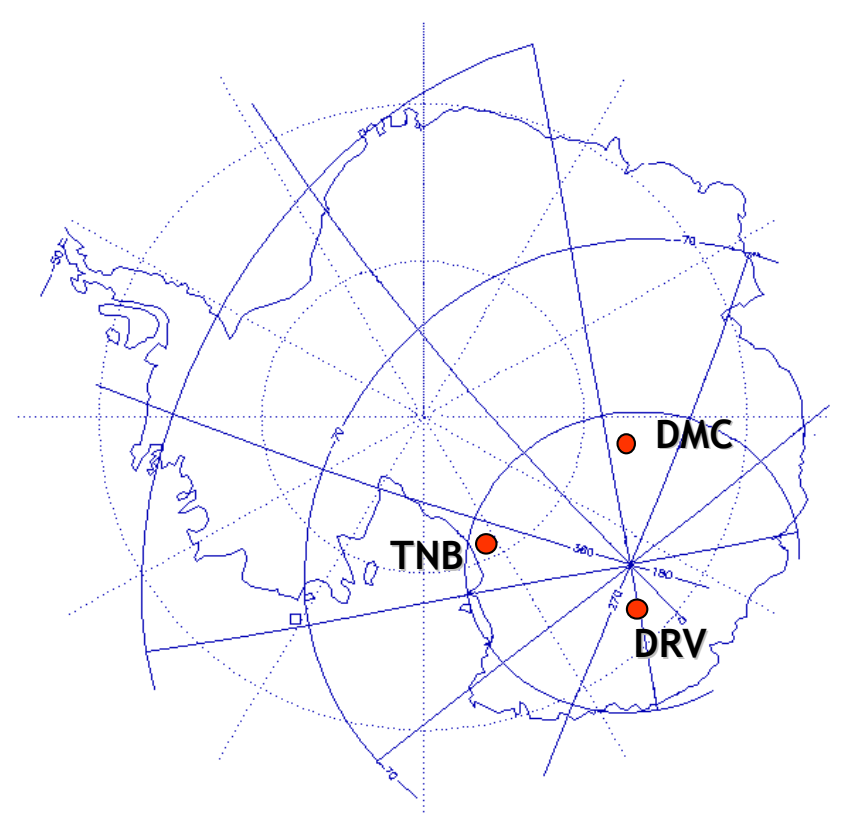

Fig. 1. The location of the three Antarctic observatories TNB, DRV and DMC, together with the geographic (dashed lines) and corrected geomagnetic (solid lines) coordinate systems over Antarctica.

The used observatories are the geomagnetic observatory at the Italian base Mario Zucchelli Station (TNB), the French Doumont D'Urville (DRV) and the French-Italian observatory at Concordia Station (DMC). The geographic and corrected geomagnetic coordinates (IGRF06) of the observatories, together with geographic local time (LT) and magnetic local time (MLT) are reported in Table 1 (corrected geomagnetic coordinates and MLT from NASA service: http: //omniweb.gsfc.nasa.gov/vitmo/cgm_vitmo.html). The relative position of the three observatories is particularly interesting in order to distinguish between geographic and geomagnetic latitude dependence of the observed phenomena in that two of them are located at the same geographic latitude (TNB and DMC) and two at the same corrected geomagnetic latitude (TNB and DRV). The location of the three Antarctic observatories TNB, DRV and DMC, together with the geographic (dashed lines) and corrected geomagnetic (solid lines) coordinate systems over Antarctica, is shown in Fig. 1. DMC is located near $89^{\circ}$ geomagnetic latitude, deep in the geomagnetic polar cap. Conversely, TNB and DRV, during periods of average magnetic activity, are inside the polar cap but quite close to the auroral oval and, under particular geomagnetic conditions and for a particular range of MLT's, they could be situated under the southern polar cusp; thus the geomagnetic variations in this condition could be heavily influenced by local cusp phenomena, mainly related to field-aligned currents (Matsushita and Xu, 1982; Campbell, 1997).

Variations in the Earth's magnetic field are continuously monitored in the three observatories by means of three-axis fluxgate magnetometers. The fundamental sampling rate is, for all the observatories, $1 \mathrm{~s}$ with a data storing rate of $1 \mathrm{~min}$. DMC and TNB fluxgate magnetometers are oriented with respect to the local magnetic meridian, so in the horizontal plane they measure the $H$ and $D$ components, directed toward the magnetic north and east directions, respectively; conversely, the DRV fluxgate magnetometer is oriented with respect to the geographic meridian, so we have the horizontal components $X$ and $Y$, directed along the geographic meridian and parallel, respectively. The angular rotation between the geographic and magnetic meridian at each station is given by the declination $D$ (in Table 1 the average $D$ values from the experimental data are reported). To compare the different data sets, a rotation of the data into an homogeneous reference system was necessary; we initially performed the analysis both in the geographic and the geomagnetic reference systems, in order to ascertain which of them gives better results in comparing the daily variation observed at the three stations. In the study of magnetospheric phenomena, especially at high latitude, MLT is an important parameter. However, MLT becomes meaningless approaching the geomagnetic pole, where it cannot be defined; in this sense, we have to keep in mind that MLT at DMC is strongly dependent on the IGRF model, so could be affected by some uncertainty.

In order to investigate the solar wind-magnetosphere interaction, we also used IMF data, in the GSM coordinate system, from ACE spacecraft (CDAWEB, http://cdaweb.gsfc. nasa.gov) and in particular the IMF component parallel to the axial dipole, $B_{z}$, and the east-west component $B_{y} . B_{z}$ is important in that positive and negative values generally 

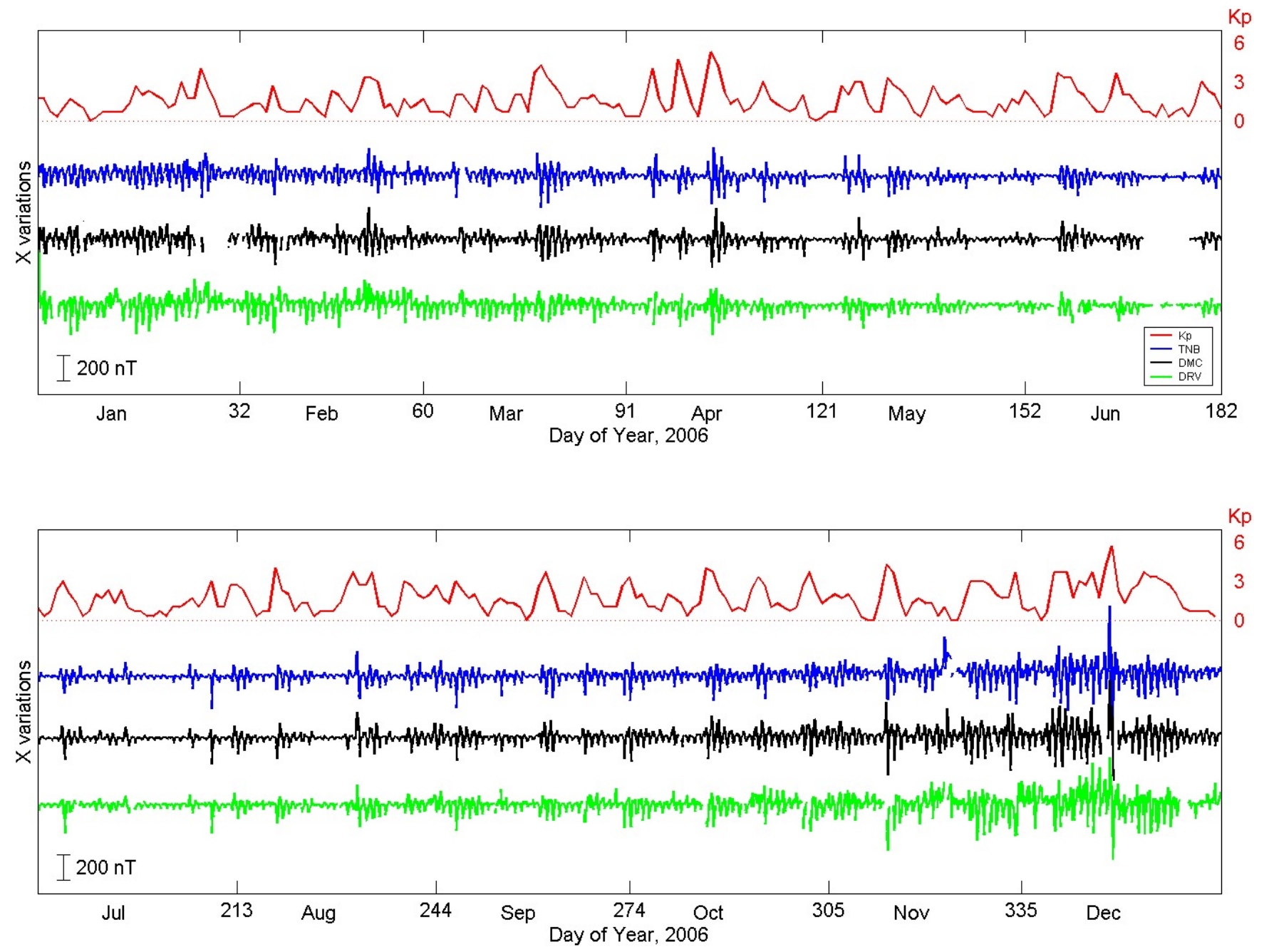

Fig. 2. Hourly values of the variations of the $\mathrm{X}$ component during 2006 for the three observatories, with the daily averages of the $K_{p}$ index.

correspond to closed or open magnetospheric conditions and, at these high latitudes, to reduced or expanded polar cap conditions, respectively (Iijima, 2000). Furthermore, also $B_{y}$ plays a main role in determining geomagnetic variations because it can create an asymmetry about the noon-midnight meridian, the well known Svalgaard-Mansurov effect (see for example McEwen, 1998; Zhou et al., 2000a; Stubbs et al., 2001). More recently the importance of $B_{y}$ for the polar cap currents also for positive $B_{z}$ conditions has been found (Vennerstrom et al., 2002; Vennerstrom et al., 2005; Park et al., 2006): the eastward IMF component, during northward IMF conditions, gradually opens the magnetosphere poleward of the cusp (Burch, 1973), changing the pattern of the ionospheric currents that become asymmetric respect to the noon-midnight meridian.

In the following we will use, as sorting parameter for different magnetospheric conditions, hourly averages of the level 2 data from CDAWEB. The total delay time from ACE and ground measurements is due to the propagation time from spacecraft position (about $235 R_{E}$ in the Earth-Sun direction) to the magnetopause (50-60 $\mathrm{min}$ ), the response time of the ionosphere (5-10 $\mathrm{min})$ and the time for the current system to develop (10-15 min) (e.g. Moretto et al., 2002; Ridley et al., 1998; Vennerstrom et al., 2002); since our analysis is based on hourly average data, we took into account a 1-h delay time.

\section{Data analysis}

In this study a daily variation analysis was performed using hourly average values of the variations of the geomagnetic field horizontal components. As an example, the hourly $X$ values during 2006 for the three observatories are shown together with the daily averages of the $K_{p}$ index in Fig. 2 (six months are reported in each box in order to have a clearer sight of the data). In this plot, for shortness, we show only the North-South component in the geographic reference system, 
TNB - X component
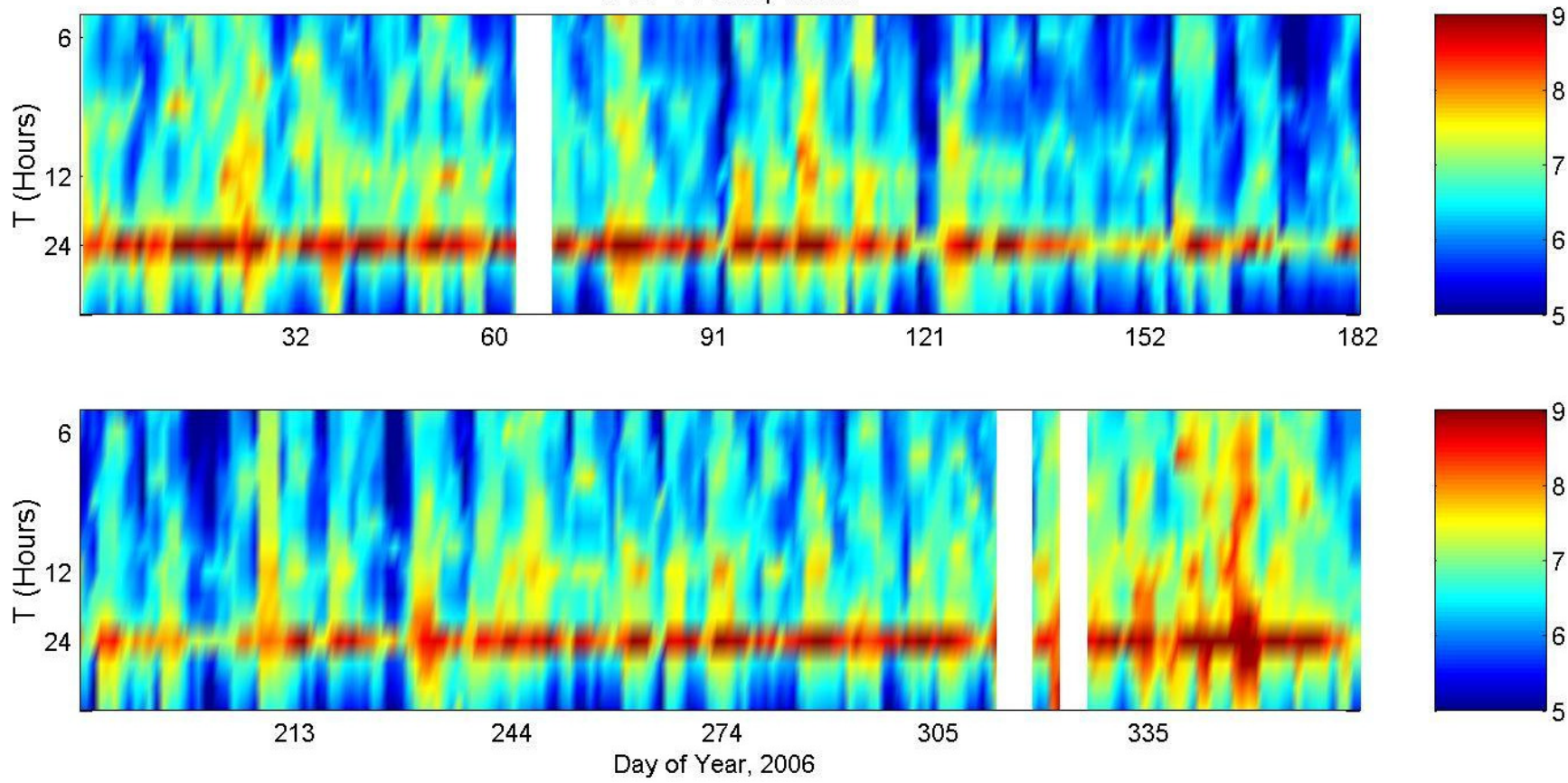

Fig. 3. Dynamic spectrum (a.u.) of the X component at TNB during 2006.

but we underline that the variations are similarly evident in both horizontal components and in both the geographic and geomagnetic reference system.

The presence of a quite regular 24-h variation is evident from the plot and it is clear that its amplitude is strongly related to $K_{p}$ values. During austral winter, for quiet geomagnetic conditions, the daily variation almost vanishes; conversely, during summer it is always present, although with a smaller amplitude, even when $K_{p}=0$. In order to investigate thoroughly the subject, we compared the spectral power of the daily variation with the $K_{p}$ index. In order to make the spectral analysis, we choose a time scale of three days, long enough to well define a 24-h signal, but not too long in order to avoid mixing very different magnetospheric conditions; then, power spectra for consecutive 72-h intervals with a step size of 24-h were computed. In Fig. 3 the dynamic spectrum for the X component at TNB is shown, as an example; it is evident that a persistent peak in correspondence of 24-h period emerges; in some cases, during local winter, its amplitude decreases, in correspondence of the lowest $K_{p}$ values (as evident from a comparison with the previous figure). As shown in Fig. 4, the logarithmic total horizontal power (sum of the power of the two horizontal components) of the 24-h peak is well related to the 3-day averages of the $K_{p}$ index (similar results are obtained considering separately the two components, both in the geographic and the geomagnetic reference system). The correlation coefficient $\rho$ for the three observatories is slightly higher during winter than during summer. Moreover, the slope of the best linear fit con- siderably increases from summer to winter: the 24-h power level for the highest $K_{p}$ values (around 3 and more) is similar in the different seasons, while for very low $K_{p}(<1)$ during winter it is of an order of magnitude lower with respect to summer. This reduction of the winter diurnal variation for quiet geomagnetic conditions is less evident at DRV, which is located at the lowest geographic latitude, where the solar radiation reduction during winter is lower. The relation between the diurnal variation amplitude and $K_{p}$ and its seasonal dependence can be attributed to the strong reduction of $S_{q}^{p}$ in the whole year during very quiet magnetospheric conditions, and even more during winter, especially at higher geographic latitude where the ionospheric ionization is more strongly reduced.

From Fig. 2 it is evident that the amplitude of the daily variation is similar at the three stations during the whole year. To get a quantitative measure of the agreement, we computed the correlation coefficient between the total horizontal 24-h power at different stations, selecting the two stations with the same geographic latitude (TNB and DMC) and the two ones with the same corrected geomagnetic latitude (TNB and DRV); the results are reported in Tables 2 and 3, respectively. In order to investigate the solar wind-magnetosphere interaction, we also considered different interplanetary conditions separately, i.e. positive and negative values of $B_{z}$ (3-days averages). Comparing the two tables, we note that the most evident difference between the two couples of stations emerges during summer, when the stations with the same geographic latitude but different corrected geomagnetic latitude have a 

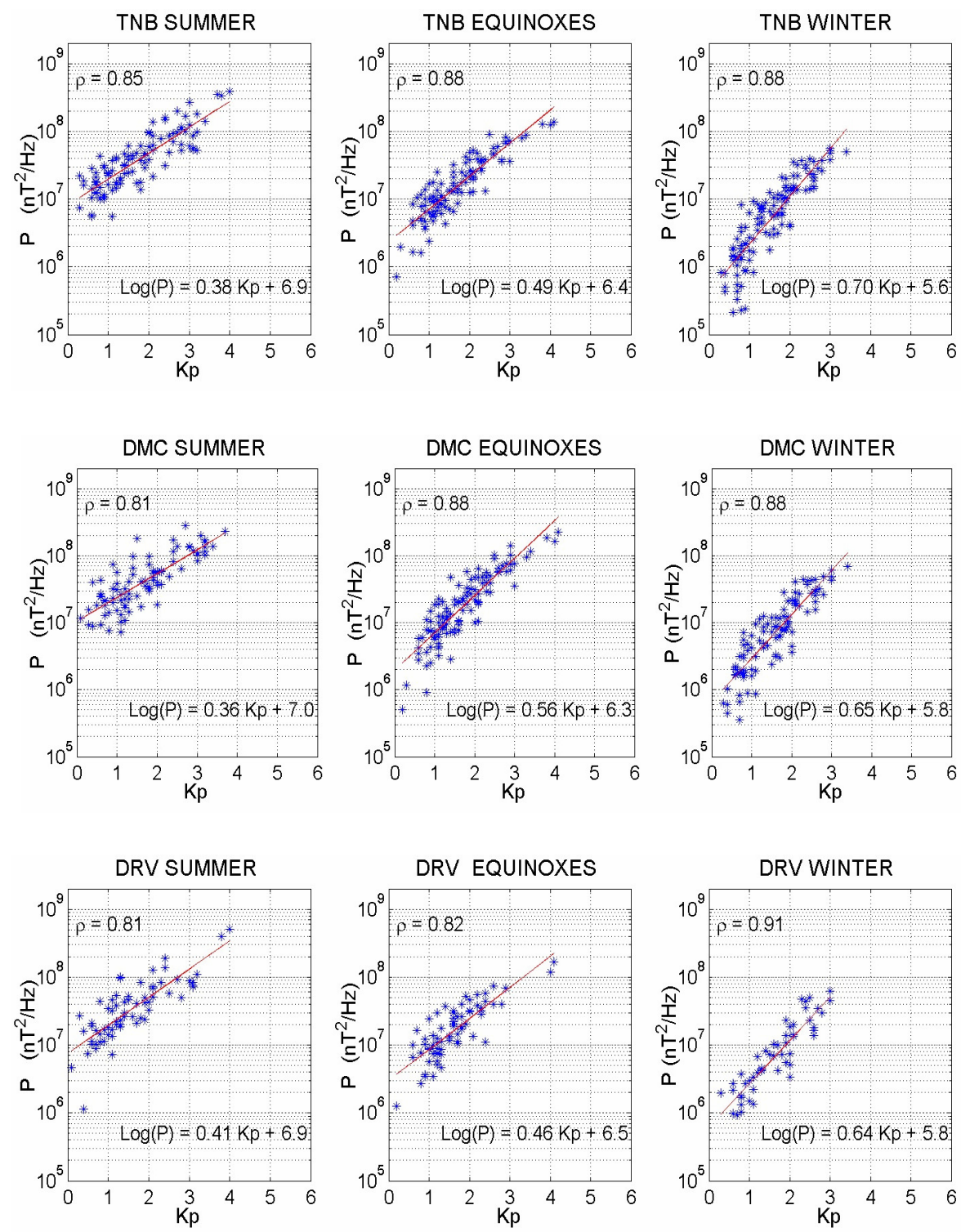

Fig. 4. The total $(\mathrm{X}+\mathrm{Y}) 24-\mathrm{h}$ power versus the $K_{p}$ index for TNB, DRV and DMC respectively. The equation of the best linear fit and the value of the correlation coefficient $\rho$ are indicated inside the boxes.

lower correlation coefficient; these results can be explained taking into account that during summer TNB and DRV approach the polar cusp (which is located at higher latitudes during local summer; Zhou et al., 1999), while DMC is always deep in the polar cap. This difference is more evident for reduced polar cap conditions ( $B_{z}>0$; Zhou et al., 2000b). An analysis performed for positive and negative $B_{y}$ values (not reported in the table) did not show any significant difference.

Next, we compare the daily distribution of the average hourly values of the two horizontal components in the geographic and the geomagnetic reference systems. In Fig. 5 the average daily variation at the three stations is shown. Each single point represents the variation at a fixed hourly aver-
Table 2. Correlation coefficient $\rho$ between the 24-h power at TNB and DMC, located at the same geographic latitude; the corresponding number of data points $N$ are given in brackets.

\begin{tabular}{lccc}
\hline DMC-TNB $\rho(\mathrm{N})$ & Summer & Equinoxes & Winter \\
\hline All & $0.75(73)$ & $0.94(109)$ & $0.99(104)$ \\
$B_{z}>0$ & $0.62(42)$ & $0.86(62)$ & $0.99(58)$ \\
$B_{z}<0$ & $0.85(30)$ & $0.98(47)$ & $0.98(46)$ \\
\hline
\end{tabular}

aged over the whole year, and the error bar is the standard deviation; the left panels refer to the geographic reference system (with LT as sorting parameter), the right panels to 

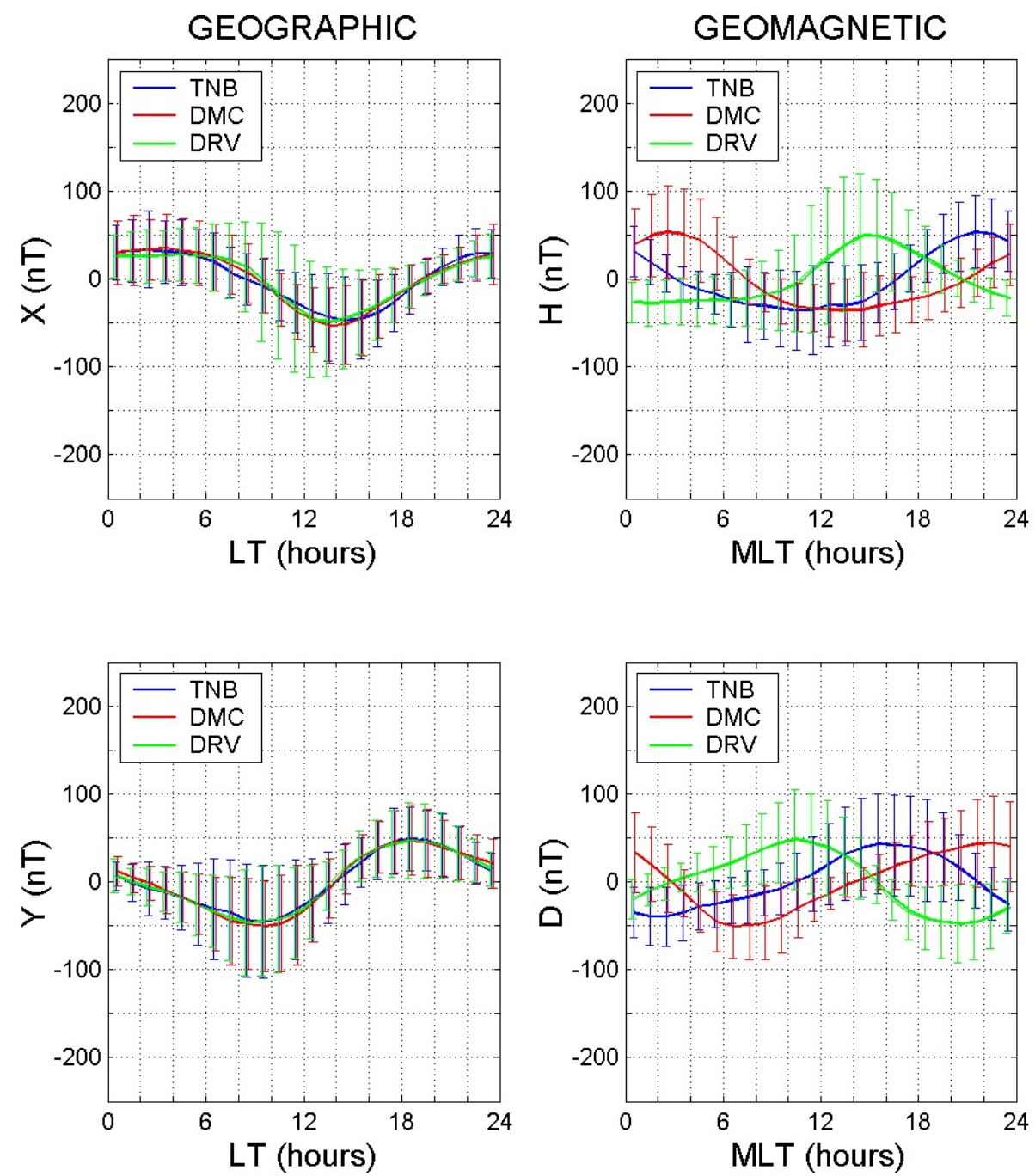

Fig. 5. Daily distribution of the average hourly values and their standard deviation in the geographic reference system (X and $\mathrm{Y}$ components, with LT as sorting parameter; left panels), and in the geomagnetic reference system ( $H$ and $D$ components, with MLT as sorting parameter; right panels).

Table 3. Correlation coefficient $\rho$ between the 24-h power at TNB and DRV, located at the same cgm latitude; the corresponding number of data points $N$ are given in brackets.

\begin{tabular}{lccc}
\hline DRV-TNB $\rho(\mathrm{N})$ & Summer & Equinoxes & Winter \\
\hline All & $0.96(57)$ & $0.96(63)$ & $0.98(54)$ \\
$B_{z}>0$ & $0.93(33)$ & $0.93(37)$ & $0.96(35)$ \\
$B_{z}<0$ & $0.97(21)$ & $0.97(26)$ & $1.00(19)$ \\
\hline
\end{tabular}

the geomagnetic reference system (with MLT). It is evident that the variation of the $\mathrm{X}$ and $\mathrm{Y}$ components shows exactly the same LT dependence and has the same amplitude at the three stations, while the variation of the $H$ and $D$ components clearly shows phase shifts between the stations.
The geographic reference system, with respect to the geomagnetic one, gives definitely better results in comparing the daily variation observed at the three stations also at these high latitudes. In the following analysis we use the two horizontal components $\mathrm{X}$ and $\mathrm{Y}$, together with LT as sorting parameter.

From the figure it is clear that, in the geographic reference system, the diurnal variation for the $\mathrm{X}$ component is symmetric around local noon with a minimum around 13:00 LT, while for $\mathrm{Y}$ it is antisymmetric, with a minimum in the local morning and a maximum in the local afternoon.

In Fig. 6 the daily distribution of the average hourly values of the $\mathrm{X}$ and $\mathrm{Y}$ components and their standard deviation for the three observatories are reported, separately for three seasons selected according to the Lloyd criteria (Lloyd, 1861): summer (November, December, January and 

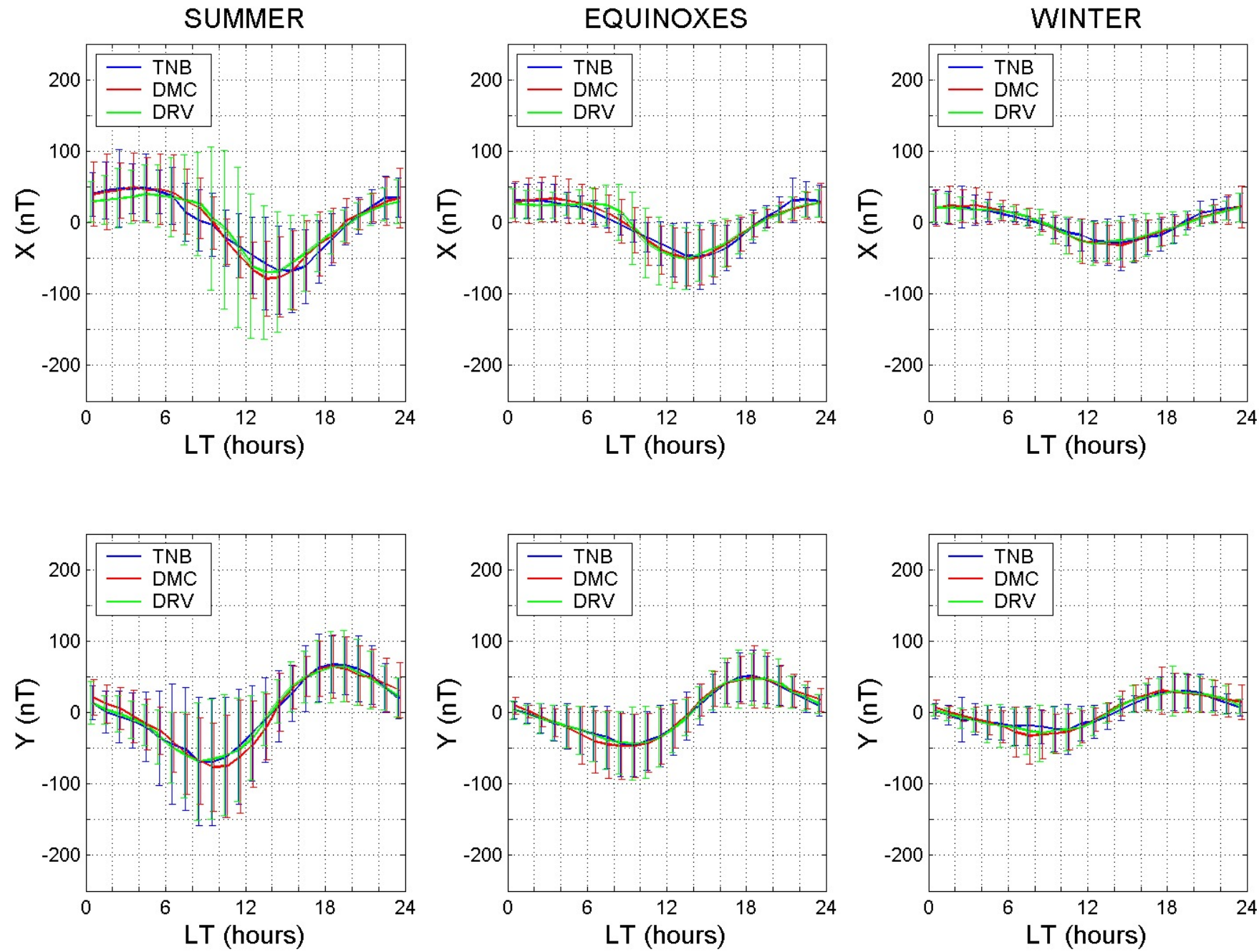

Fig. 6. Daily distribution of the average hourly values and their standard deviation for the $\mathrm{X}$ and $\mathrm{Y}$ components, considering separately the three Lloyd seasons.

February), equinoxes (March, April, September and October) and winter (May, June, July and August). From the figure it is clear that the general pattern of the diurnal variation does not show a seasonal dependence. Conversely, its amplitude depends on season, being larger during local summer (with a mean peak-to-peak amplitude exceeding $100 \mathrm{nT}$ ) and smaller during winter (around 50 nT). Also the amplitude of the error bars is larger during local summer, indicating a stronger day-to-day variability. Comparing the daily variation at the three stations, we can see that it is almost identical during equinoxes and winter, while some differences emerge during summer, when there is a slight time shift. Additionally, during daytime hours, a difference in the amplitude of the error bars can be observed. They are smaller for DMC, i.e. for the station which does not approach the polar cusp and larger for DRV.

Finally, we considered different IMF conditions. First of all we computed the daily variation pattern for positive and negative values of $B_{z}$; the results are shown in Fig. 7. From the comparison of the plots it is evident that the general pattern of the diurnal variation does not depend on $B_{z}$ conditions, while the amplitude of the variation is definitively larger for southward $\left(B_{z}<0\right)$ than for northward $\left(B_{z}>0\right)$ directed IMF; the standard deviation, too, takes on larger values in the condition of $B_{z}<0$. In both cases the pattern of diurnal variation is almost identical among the three stations, independently on $B_{z}$ conditions. Also considering separately different seasons (not shown here) no additional difference, respect to the analysis reported in Fig. 6, emerges.

In Fig. 8 the diurnal variation at each observatory is reported, separately for positive and negative $B_{y}$ conditions. The results show that the different $B_{y}$ conditions correspond to different features at the three stations. At TNB minor differences in the two different $B_{y}$ conditions emerge: just a slight amplitude variation (larger for positive $B_{y}$ ) and time shift (earlier for negative $B_{y}$ ); for DMC the diurnal variation 

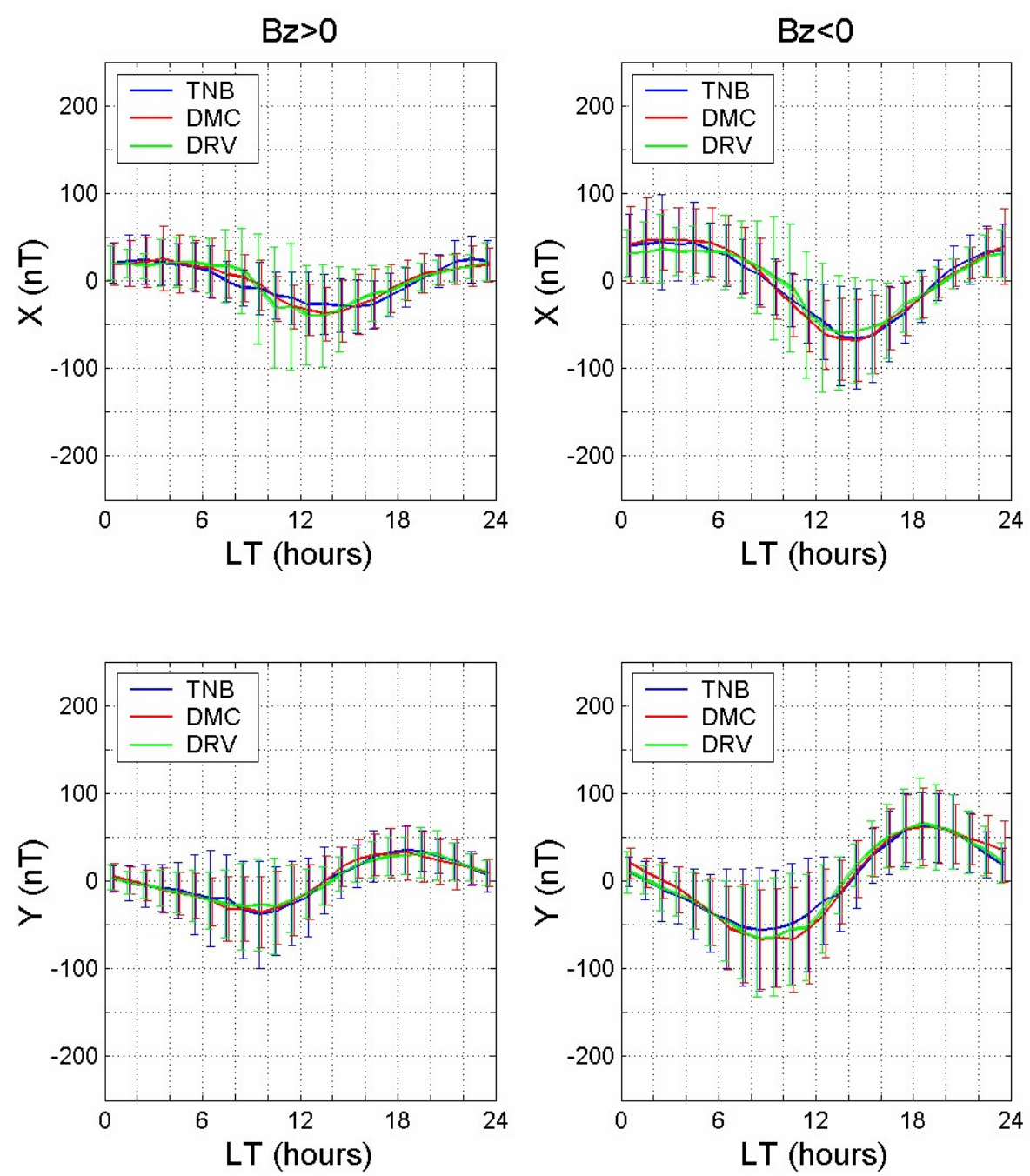

Fig. 7. Daily distribution of the average hourly values and their standard deviation for the $\mathrm{X}$ and $\mathrm{Y}$ components, separately for northward (left panels) and southward (right panels) IMF conditions.

for negative $B_{y}$ is larger and is clearly shifted to earlier times by about $3 \mathrm{~h}$ with respect to positive $B_{y}$; lastly, for DRV the daily variation for negative $B_{y}$ is smaller and has an opposite time shift for the two components: later for $X$ and earlier for $Y$.

We also performed the analysis considering separately positive and negative conditions both for $B_{y}$ and $B_{z}$; in Fig. 9 we compare the daily variation at the three observatories for each different IMF orientation. It can be seen that the most evident differences between the stations emerge for negative $B_{y}$; in this conditions, indeed, the pattern of the diurnal variation at DRV is quite different, especially for reduced polar cap conditions $\left(B_{z}<0\right)$.

\section{Summary and conclusions}

In this paper we present the analysis of 2006 data coming from three different Antarctic geomagnetic observatories located in different positions within the polar cap. In this work, the considered observatories are TNB, DRV and DMC. Their position allows to investigate the latitude dependence of diurnal variation and its representation in geographic and geomagnetic reference systems; in fact they are located in pairs at the same geographic and corrected geomagnetic latitude, respectively.

The daily variation has been analysed for the whole 2006, an year of low geomagnetic activity. The very first result was the presence of a quite regular 24-h variation and it was shown that its amplitude is strongly related to $K_{p}$. The correlation between these two quantities is particularly significative during winter, when, especially at stations at higher 
TNB
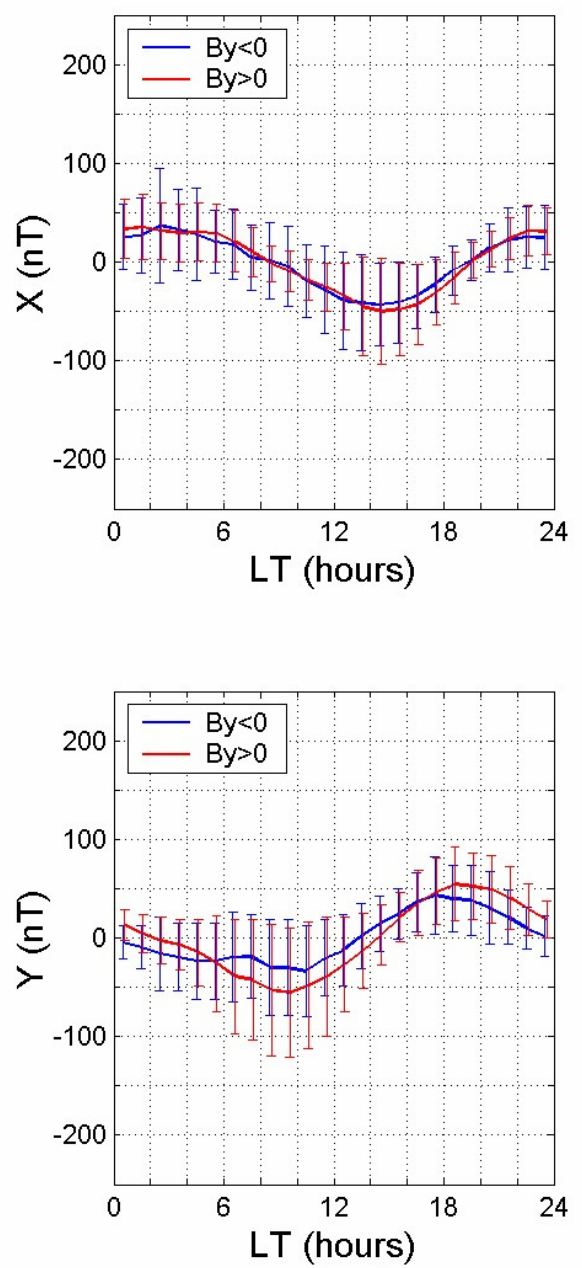

DMC
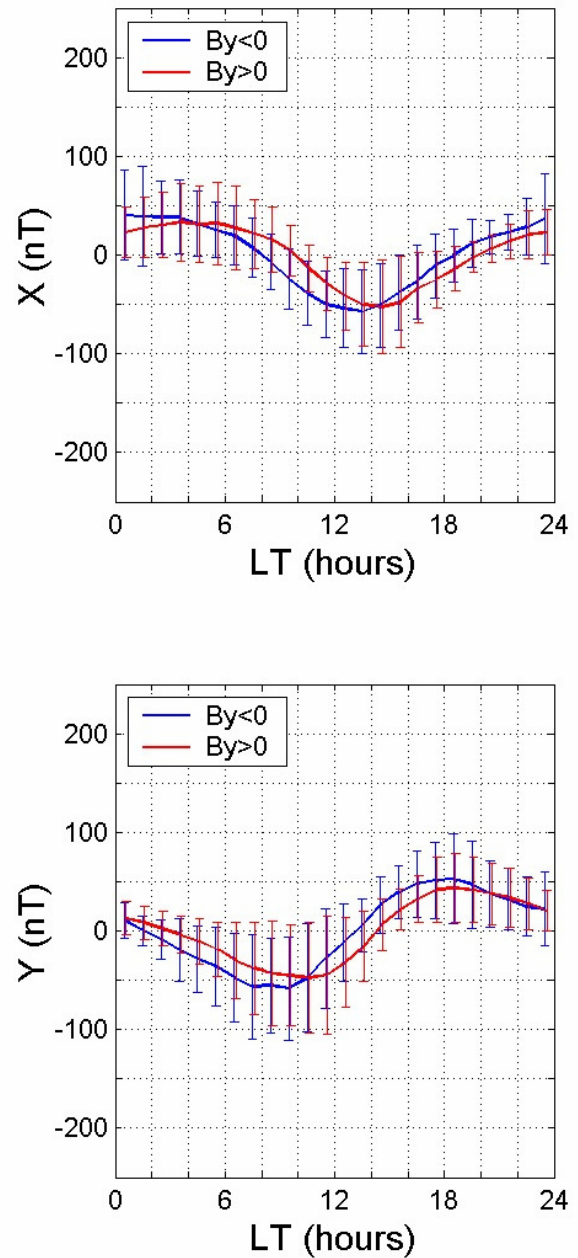

DRV
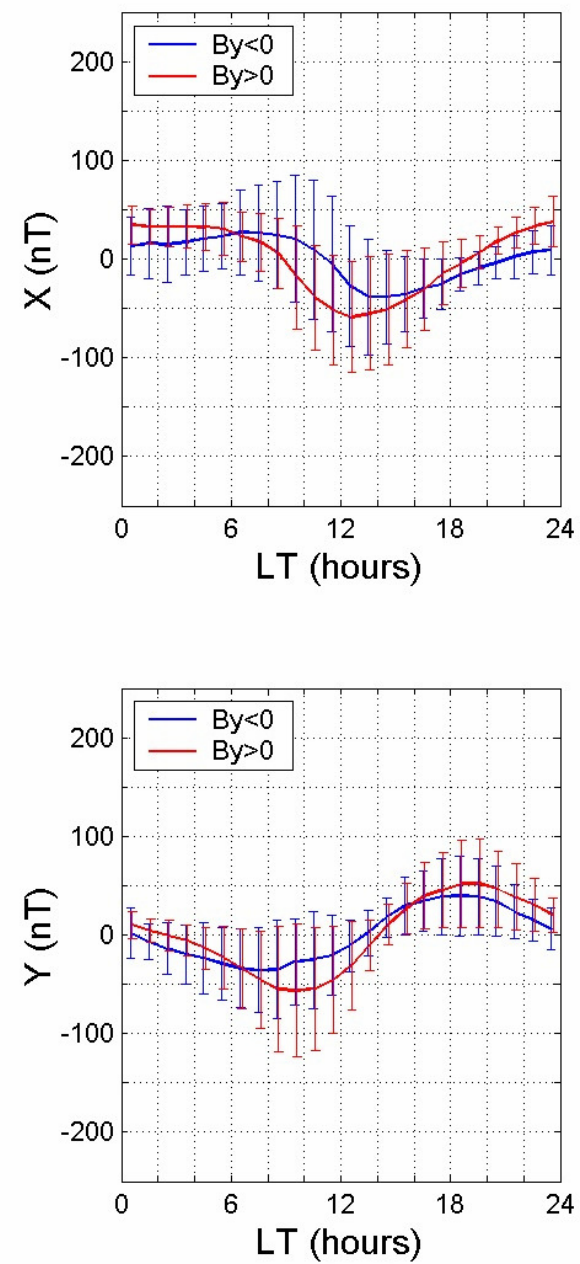

Fig. 8. Daily distribution of the average hourly values and their standard deviation for the $\mathrm{X}$ and $\mathrm{Y}$ components, separately for positive and negative $B_{y}$.

geographic latitude, there is a dramatic amplitude attenuation for quiet magnetospheric conditions, ascribable to the strong reduction of $S_{q}^{p}$ currents; this is due to the ionospheric ionization at these high latitudes also linked to the complete absence of solar radiation.

We analyzed the correlation between the amplitude of the 24-h variation at the two couples of stations with the same geographic (TNB and DMC) and corrected geomagnetic (TNB and DRV) latitude, also selecting different interplanetary conditions (positive and negative values of the component parallel to the axial dipole, $B_{z}$, and the east-west component $B_{y}$ ). This analysis showed that an evident difference emerges during summer for reduced polar cap conditions $\left(B_{z}>0\right)$, when the amplitude of the daily variation is less correlated between the two stations at different corrected geomagnetic latitude (TNB and DMC). This results can be explained taking into account that the polar cusp position shifts poleward for closed magnetospheric conditions and during local summer (Staisiewicz, 1991; Iijima, 2000; Zhou et al., 1999, 2000b), then in these conditions TNB and DRV roughly approach the polar cusp, while DMC is always deep in the polar cap. The stations are so located in different magnetospheric regions, and TNB and DRV can experiment geomagnetic phenomena specific of the auroral oval, which are decoupled from simultaneous observations in the polar cap (Yagova et al., 2002, 2004). The analysis performed for positive and negative $B_{y}$ values (not reported in the tables) did not show any significant difference.

For the analysis of the diurnal pattern of the daily variation, we started with the question whether the best reference system for ordering this phenomenon at cap latitudes is the geographic or the corrected geomagnetic one (with LT and MLT as ordering parameter, respectively). We found that the geographic reference system is better suitable to compare the daily variation observed at the three stations; indeed, considering the diurnal variation of the horizontal $\mathrm{X}$ and $\mathrm{Y}$ 

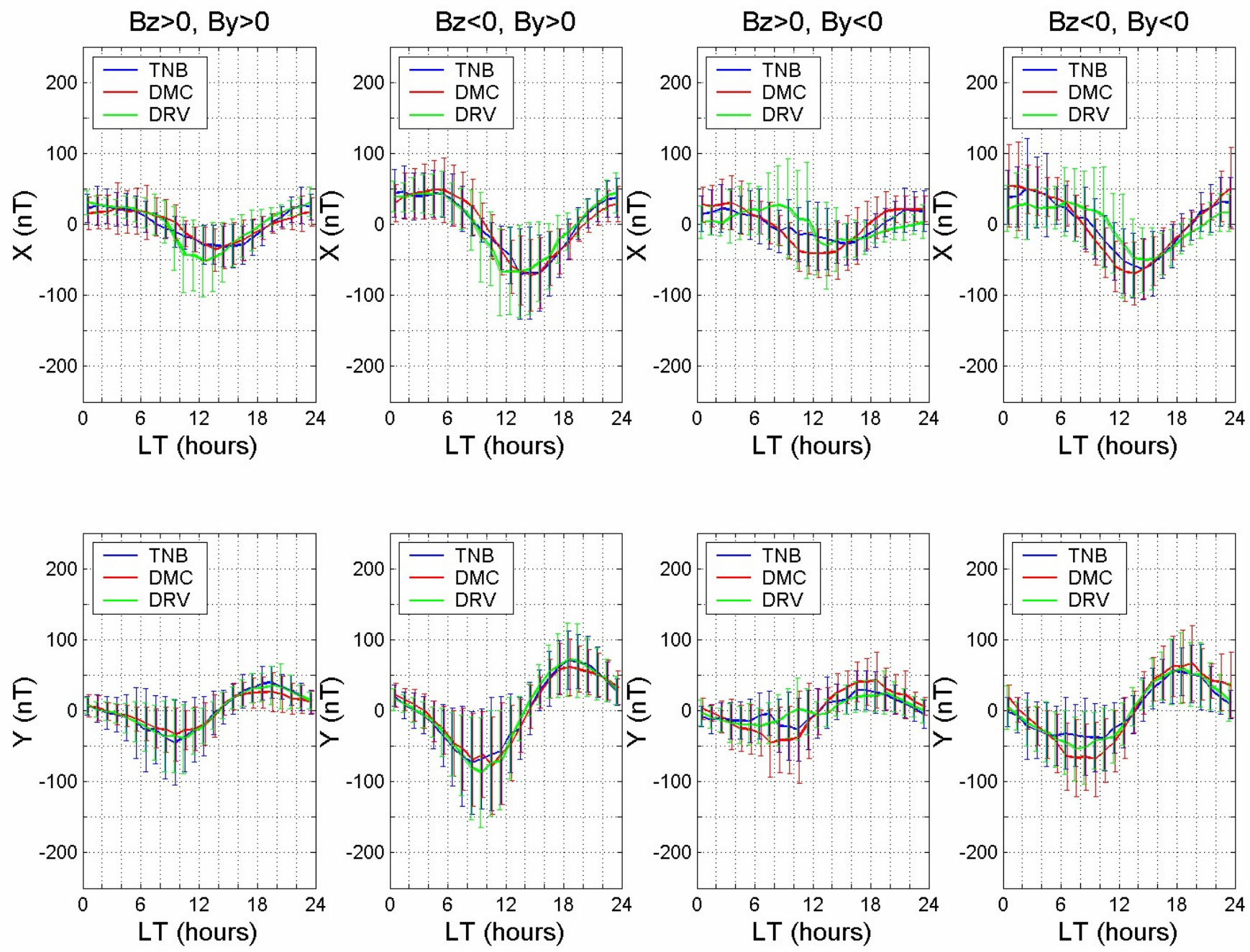

Fig. 9. Daily distribution of the average hourly values and their standard deviation for the $\mathrm{X}$ and $\mathrm{Y}$ components, separately for positive and negative conditions of both $B_{y}$ and $B_{z}$.

components versus LT, we obtain an almost identical diurnal pattern at the three stations.

The analysis performed separately for three Lloyd seasons did not show any evident difference between the three observatories; for all of them the diurnal pattern is not dependent on season, while the amplitude decreases from local summer to winter. Even considering different conditions of the northsouth IMF component, $B_{z}$, corresponding to open or closed magnetosphere and to expanded or reduced polar cap (Iijima, 2000), there is no difference in the daily variation observed at the three observatories and in any case the amplitude of the daily variation is higher for negative $B_{z}$.

Also the east-west IMF component, $B_{y}$, important in determining geomagnetic variations at high latitudes, was considered. In general, for negative values of $B_{y}$, the magnetic activity in the high latitude Southern Hemisphere tends to be higher (Nishida, 1978). Moreover, when $B_{z}$ assumes negative values, the $B_{y}$ component creates an asymmetry about the noon-midnight meridian in the evolution of newly opened field lines (Svalgaard-Mansurov effect; see for example McEwen, 1998; Zhou et al., 2000a; Stubbs et al., 2001). More recently, $B_{y}$ has been shown to be important also for positive $B_{z}$ conditions, when the existence and the geometry of the polar cap, as well as the local current geometry, is strongly connected to the value of the $B_{y}$ component (Vennerstrom et al., 2002, 2005; Park et al., 2006): the introduction of an eastward IMF component gradually opens the magnetosphere poleward of the cusp (as originally suggested by Burch, 1973), with newly open field lines which in the Northern Hemisphere are displaced toward dawn (dusk) for positive (negative) $B_{y}$ (the contrary in the Southern Hemisphere). This process changes also the pattern of the ionospheric currents that are then located asymmetrically with respect to the noon-midnight meridian; in particular, in the Southern Hemisphere, dusk (dawn) side currents are enhanced for positive (negative) $B_{y}$ (Vennerstrom et al., 2005). 
Consistent with this scenario, our analysis has shown that at DMC, i.e. close to the geomagnetic pole, the diurnal variation for negative $B_{y}$ is larger and the curve is shifted to earlier times by about $3 \mathrm{~h}$ with respect to positive $B_{y}$ (see also Cafarella et al., 2007). It is interesting that these features do not appear at the stations at lower geomagnetic latitude (there is just some tendency to a slight time shift, more evident for the Y component, at TNB), indicating that the IMF orientation contributes differently at various positions within the polar cap. We also found that the diurnal variation at DRV, for $B_{y}<0$ has a peculiar shape with respect to the other stations, and this feature is more evident for reduced polar cap conditions; the anomalous behaviour emerges in the morning hours, when the ionospheric currents are expected to be enhanced in $B_{y}<0$ conditions (Vennerstrom et al., 2005); the appearance of this feature only at the lowest geographic latitude station could be related to the higher solar radiation, and then to the higher ionospheric ionization.

We would like to conclude this brief paper underlining that the characteristics of the daily variation, also at such high latitudes, seem to be better described in a geographic coordinate system rather than in a geomagnetic one This suggests that in the analyzed time period the contribution of the $S_{q}^{p}$ polar electric current systems are not very relevant. These results are partially justified by the fact that the study has been performed for one year at the minimum of the present solar cycle. We can conclude that under these conditions the mid latitude ionospheric currents seem to be the main source of the daily variation.

Acknowledgements. The research activity at TNB is supported by Italian PNRA (Programma Nazionale di Ricerche in Antartide). The research activity at DMC is supported by Italian PNRA and French IPEV (Institut Polaire Français Paul Emile Victor). The research activity at DRV is supported by French IPEV (Institut Polaire Français Paul Emile Victor). The work of M. Pietrolungo and L. Santarelli was supported by a PNRA fellowship.

The authors thank J. J. Schott (Ecole et Observatoire des Sciences de la Terre, France) that operates the INTERMAGNET observatory DRV. ACE data were provided by CDAWeb, run by NASA GSFC; thanks also to the ACE team and the ACE Science Center.

Topical Editor I. A. Daglis thanks P. Ritter and another anonymous referee for their help in evaluating this paper.

\section{References}

Akasofu, S. I., Ahn, B. H., and Romick, G. J.: A study of the polar current system using the IMS meridian chains of magnetometers, Space Sci. Rev., 36, 337-413, 1983.

Brekke, A.: Physics of the upper polar atmosphere, John Wiley \& Sons Ltd in association with Praxis Publishing Ltd, p. 491, 1997.

Burch, J. L.: Rate of erosion of dayside magnetic flux based on a quantitative study of polar cusp latitude on the interplanetary magnetic field, Radio Sci., 8, 955-961, 1973.

Cafarella, L., Di Mauro, D., Lepidi, S., Meloni, A., Pietrolungo, M., Santarelli, L., and Schott, J. J.: Daily variation at Concordia station (Antarctica) and its dependence on IMF conditions, Ann. Geophys., 25, 2045-2051, 2007,

http://www.ann-geophys.net/25/2045/2007/.

Campbell, W. H.: Introduction to geomagnetic fields, Cambridge University Press, 135-142, 1997.

Kivelson, M. G. and Russell, C. T.: Introduction to Space Physics, Cambridge University Press, p. 568, 1996.

Iijima, T.: Field-aligned currents in geospace: substance and significance, Magnetospheric Current Systems, edited by: Ohtani, S.-I., Fujii, R., Hesse, M., and Lysak, R. L., Gephysical Monograph, 118, 107-129, 2000.

Lepidi, S., Cafarella, L., Francia, P., Meloni, A., Palangio, P., and Schott, J. J.: Low frequency geomagnetic field variations at Dome C (Antartica), Ann. Geophys., 21, 923-932, 2003, http://www.ann-geophys.net/21/923/2003/.

Lloyd, H.: On earth-currents, and their connexion with the diurnal changes of the horizontal magnetic needle, Trans. Roy. Irish Acad., 24, 115-141, 1861.

Matsushita, S. and Xu, W.: Equivalent ionospheric current system representing solar daily variations of the polar geomagnetic field, J. Geophys. Res., 87, 8241-8254, 1982.

McEwen, D. J.: Polar cap phenomena and their relation to boundary layers and the IMF, in: Polar cap Boundary Phenomena, edited by: Moen, J., Egeland, A., and Lockwood, M., 271-280, 1998.

Moretto, T., Olsen, N., Ritter, P., and Lu, G.: Investigating the auroral electrojets with low altitude polar orbiting satellites, Ann. Geophys., 20, 1049-1061, 2002, http://www.ann-geophys.net/20/1049/2002/.

Nishida, A.: Geomagnetic diagnosis of the magnetosphere, Springer-Verlag, 54-64, 1978.

Park, K. S., Ogino, T., and Walker, R. J.: On the importance of antiparallel reconnection when the dipole tilt and IMF $B_{y}$ are nonzero, J. Geophys. Res., 111, A05202, doi:10.1029/2004JA010972, 2006.

Ratcliffe, J. A.: An introduction to the ionosphere and magnetosphere, Com. Uni Press, Cambridge, p. 256, 1972.

Ridley, A. J., Gang, Lu, Clauer, C. R., and Papitashvili, V. O.: A statistical study of the ionospheric convection response to changing interplanetary magnetic field conditions using the assimilative mapping of ionospheric electrodynamics technique, J. Geophys. Res., 103(A3), 4023-4039, 1998.

Santarelli, L., Cafarella, L., Lepidi, S., Di Mauro, D., Meloni, A., and Palangio, P.: Fourteen years of geomagnetic daily variation at Mario Zucchelli Station (Antarctica), Annals of Geophysics, Italy, 50, 225-232, 2007.

Stasiewicz, K.: Polar cusp topology and position as a function of interplanetary magnetic field and magnetic activity: Comparison of a model with Viking and other observations, J. Geophys. Res., 96, 15 789-15 800, 1991.

Stubbs, T. J., Lockwood, M., Cargill, P., Fennell, J., Grande, M., Kellett, B., Perry, C., and Rees, A.: Dawn-dusk asymmetry in particles of solar wind origin within the magnetosphere, Ann. Geophys., 19, 1-9, 2001, http://www.ann-geophys.net/19/1/2001/.

Vennerstrom, S., Moretto, T., Olsen, N., Friis-Christensen, E., and Stampe, A. M.: Field aligned currents in the dayside cusp and polar cap region during northward IMF, J. Geophys. Res., 107, A009162, doi:10.1029/2001JA009162, 2002.

Vennerstrom, S., Moretto, T., Rastatter, L., and Raeder, J.: 
Field-aligned currents during northward interplanetary magnetic field: Morphology and causes, J. Geophys. Res., 110, A06205, doi:10.1029/2004JA010802, 2005.

Yagova, N. V., Lanzerotti, L. J., Villante, U., Pilipenko, V. A., Lepidi, S., Francia, P., Papitashvili, V. O., and Rodger, A. S.: ULF Pc5-6 magnetic activity in the polar cap as observed along a geomagnetic meridian in Antarctica, J. Geophys. Res., 107, SMP22, doi:10.1029/2001JA900143, 2002.

Yagova, N. V., Pilipenko, V. A., Lanzerotti, L. J., Engebretson, M. J., Rodger, A. S., Lepidi S., and Papitashvili, V. O.: Two-dimensional structure of long-period pulsations at polar latitudes in Antarctica, J. Geophys. Res., 109, A03222, doi:10.1029/2003JA010166, 2004.
Zhou, X. W., Russell, C. T., Le, G., Fuselier, S. A., and Scudder, J. D.: The polar cusp location and its dependence on dipole tilt, Geophys. Res. Lett., 26, 429-432, 1999.

Zhou, X. W., Russell, C. T., and Le, G.: Local time and interplanetary magnetic field By dependence of field-aligned currents at high altitudes, J. Geophys. Res., 105, 2533-2539, 2000a.

Zhou, X. W., Russell, C. T., Le, G., Fuselier, S. A., and Scudder, J. D.: Solar wind control of the polar cusp at high altitude, J. Geophys. Res., 105, 245-251, doi:10.1029/1999JA900412, $2000 \mathrm{~b}$. 